\title{
Analysis of the Stability and Hopf Bifurcation of Currency Supply Delay in an Opened Kaldorian Business Cycle Model
}

\author{
Liming Zhao and Zhipei Zhao \\ Department of Management and Economics, Tianjin University, Tianjin 300072, China \\ Correspondence should be addressed to Zhipei Zhao; zhaozp@tju.edu.cn
}

Received 27 February 2016; Revised 15 June 2016; Accepted 14 July 2016

Academic Editor: Rosana Rodriguez-Lopez

Copyright ( 2016 L. Zhao and Z. Zhao. This is an open access article distributed under the Creative Commons Attribution License, which permits unrestricted use, distribution, and reproduction in any medium, provided the original work is properly cited.

\begin{abstract}
First of all, we establish a three-dimension open Kaldorian business cycle model under the condition of the fixed exchange rate. Secondly, with regard to the model, we discuss the existence of equilibrium point and the stability of the system near it with a time delay in currency supply as the bifurcating parameters of the system. Thirdly, we discuss the existence of Hopf bifurcation and investigate the stability of periodic solution generated by the Hopf bifurcation; then the direction of the Hopf bifurcation is given. Finally, a numerical simulation is given to confirm the theoretical results. This paper plays an important role in theoretical researching on the model of business cycle, and it is crucial for decision-maker to formulate the macroeconomic policies with the conclusions of this paper.
\end{abstract}

\section{Introduction}

Economy is a pivotal factor in the national development, and it not only closely links to the daily life of singular people but also plays an important role in maintaining the stability of society. Because of the complexity and the instability of the economic system operation, any tiny external condition changes may give rise to instability of the whole system. In recent years, more and more economists put their eyes on the field of nonlinear dynamic system and use it to build relevant economic models and do some research on the operation law of the economic system [1-3]. As is well known, a time delay always exists in most of economic systems. Therefore, as a branch of nonlinear dynamics, the delayed nonlinear dynamics have been used widely by economists. Kaldor used ordinary differential equations to research the business cycle model, and he proposed that nonlinear investment function and saving function were an important factor to generate periodical fluctuation in economic system [4]. His research had given a new direction in business cycle research. Kalecki [5] proposed a new viewpoint in researching the business cycle: the capital for operation is related to the time. In other words, original part of the investment capital is the profit saving in a period of time previously; the investment decision will influence the capital stock [6], and a period or a time delay always exists in capital investment for production in economic operational process. After summing up the idea of Kaldor and Kalecki, Krawiec and Szydlowski [7] bring about a time delay in Kaldor model and get the Kaldor-Kalecki model which shows that

$$
\begin{aligned}
& Y^{\prime}(t)=\alpha(I(Y(t), K(t))-S(Y(t), K(t))), \\
& K^{\prime}(t)=I(Y(t-\tau), K(t))-d K(t) .
\end{aligned}
$$

Reference [8], in allusion to the model above, did some research on the influences of the time delay on the stability of the system and analyzed the generation condition of the Hopf bifurcation. Kaddar and Talibi Alaoui [9] thought that investment decision would exert an influence on both the gross value of product and capital stock. They modified the second equation in (1) and transfer the investment function $I(Y(t-\tau), K(t))$ into $I(Y(t-\tau), K(t-\tau))$; that is,

$$
\begin{aligned}
Y^{\prime}(t) & =\alpha(I(Y(t), K(t))-S(Y(t), K(t))), \\
K^{\prime}(t) & =I(Y(t-\tau), K(t-\tau))-d K(t) .
\end{aligned}
$$

Reference [10] puts the time delay in Kaldor-Kalecki model and did some research on its dynamic properties. Wang and $\mathrm{Wu}$ found that the time delay would destroy the 
stability of the system and concluded that the variation of capital stock caused by investment decision was a factor of leading the economic system to an instable status.

Most of researches on Kaldor-Kalecki model, from the viewpoint of the functional differential equation or the viewpoint of the ordinary differential equation, concentrate on the enclosed type of economic system. Therefore, we will focus on an open Kaldorian business model following the research method and conclusion of the Kaldor-Kalecki model $[11,12]$. Kaldorian business cycle theory is based on the initial thoughts of Kaldor and was enriched by Lorenz [13], Gandolfo [14], and Agliari et al. [15]. According to [16], the Kaldorian model can be written in the following form:

$$
\begin{aligned}
& \frac{d Y(t)}{d t}=\alpha\left[(c(1-\varepsilon)-1) Y(t)+\left(\gamma-\beta_{1} \delta\right) \sqrt{Y(t)}\right. \\
& \left.-d_{1} K(t)+\beta_{1} \beta_{2} M(t)+c T_{0}+C_{0}+G\right], \\
& \frac{d K(t)}{d t}=\left(\gamma-\beta_{1} \delta\right) \sqrt{Y(t)}-d_{1} K(t)+\beta_{1} \beta_{2} M(t), \\
& \frac{d M(t)}{d t}=\delta_{1} Y+\beta\left(\delta \sqrt{Y(t)}-\beta_{2} M-r_{0}\right),
\end{aligned}
$$

where $Y$ is actual gross domestic product, $C$ is actual consumption expenditure, $K$ is physical capital stock, $T$ is actual income tax, $G$ is government expenditure, $M$ is nominal currency supply, $\alpha$ is the market adjustment coefficient, and $\beta$ is the degree of capital movement.

Furthermore, we denote $m=c(1-\varepsilon)-1, n=\gamma-\beta_{1} \delta$, $b=\beta_{1} \beta_{2}$, and $a=c T_{0}+C_{0}+G$; then the model can be transformed to

$$
\begin{aligned}
& \frac{d Y(t)}{d t} \\
& \quad=\alpha\left(m Y(t)+n \sqrt{Y(t)}-d_{1} K(t)+b M(t)+a\right), \\
& \frac{d K(t)}{d t}=n \sqrt{Y(t)}-d_{1} K(t)+b M(t), \\
& \frac{d M(t)}{d t}=\delta_{1} Y(t)+\beta\left(\delta \sqrt{Y(t)}-\beta_{2} M(t)-r_{0}\right) .
\end{aligned}
$$

Considering that the right-hand sides of (4) should be analytical, we bring about the transform $\sqrt{Y} \rightarrow Y$, and the system is $C^{1}(0,+\infty)$ class which is shown as

$$
\begin{aligned}
& \frac{d Y(t)}{d t}=\frac{\alpha}{2}\left(m Y(t)+n-d_{1} K(t) Y^{-1}(t)\right. \\
& \left.\quad+b M(t) Y^{-1}(t)+a Y^{-1}(t)\right), \\
& \frac{d K(t)}{d t}=n Y(t)-d_{1} K(t)+b M(t), \\
& \frac{d M(t)}{d t}=\delta_{1} Y^{2}(t)+\beta\left(\delta Y(t)-\beta_{2} M(t)-r_{0}\right)+c .
\end{aligned}
$$

The time delay of money supply is a crucial factor which influences the effects of monetary policy. Monetary policy changes normally take a certain amount of time to have an effect on the economy. The time delay could span anywhere from nine months up to two years. Fiscal policy and its effects on output have a shorter time delay. When monetary policy attempts to stimulate the economy by lowering interest rates, it may take up to 18 months for evidence of any improvement in economic conditions to show up. However, there are few papers considering the macroeconomic model with currency supply delay. In this paper, we propose that there is a finite delay between the rate and the currency supply in a frame work. We study how the delay affects the macroeconomic stability. By using the theory of normal and center manifold theorem, an explicit algorithm for determining the direction of Hopf bifurcation and the stability of bifurcating periodic solutions is derived. To the best of our knowledge, this paper is the first one to deal with Hopf bifurcation about the dynamic Kaldor-Kalecki model with discrete delay in currency supply. Considering currency supply delay to macroeconomic model is very important to implement the monetary policy and the government's stabilization policy.

This paper is organized as follows. In Section 2, we investigate the local stability of the equilibrium point of system (6). By choosing the delay as the bifurcation parameter, some sufficient conditions for the existence of Hopf bifurcation are found. In Section 3, the formulas determining the direction and the stability of the bifurcating periodic solutions are obtained by the normal form theory and center manifold theorem introduced by Hassard et al. [17]. In Section 4, some numerical simulations consequences are given. Finally, some conclusions are made.

\section{Equilibrium Stability and Existence of the Hopf Bifurcation}

Bring the time delay in currency supply; system (5) becomes

$$
\begin{aligned}
& \frac{d Y(t)}{d t}=\frac{\alpha}{2}\left(m Y(t)+n-d_{1} K(t) Y^{-1}(t)\right. \\
& \left.\quad+b M(t-\tau) Y^{-1}(t)+a Y^{-1}(t)\right), \\
& \frac{d K(t)}{d t}=n Y(t)-d_{1} K(t)+b M(t), \\
& \frac{d M(t)}{d t}=\delta_{1} Y^{2}(t)+\beta\left(\delta Y(t)-\beta_{2} M(t)-r_{0}\right)+c .
\end{aligned}
$$

At first, we will find the equilibrium of system (6) with $\tau=0$, and the equilibrium point is equal to the solutions of the equation sets:

$$
\begin{aligned}
& \alpha\left(m Y(t)+n-d_{1} K(t) Y^{-1}(t)+b M(t-\tau) Y^{-1}(t)\right. \\
& \left.\quad+a Y^{-1}(t)\right)=0 \\
& n Y(t)-d_{1} K(t)+b M(t)=0 \\
& \delta_{1} Y^{2}(t)+\beta\left(\delta Y(t)-\beta_{2} M(t)-r_{0}\right)+c=0
\end{aligned}
$$


Then, the equilibrium of system (6) is $E\left(Y^{*}, K^{*}, M^{*}\right)$, where

$$
\begin{aligned}
Y^{*} & =Y_{0}=\sqrt{-\frac{2 a}{m}}=\sqrt{\frac{2 c T_{0}+2 C_{0}+2 G}{1-c(1-\varepsilon)}}, \\
K^{*} & =\frac{b \beta \delta m Y_{0}+\beta m n \beta_{2} Y_{0}-b \beta m r_{0}-a b \delta_{1}+b c m}{2 m \beta \beta_{2} d_{1}}, \\
M^{*} & =\frac{\beta \delta m Y_{0}-\beta m r_{0}-a \delta_{1}+c m}{2 m \beta \beta_{2}} .
\end{aligned}
$$

Through the linear transformation,

$$
\begin{aligned}
& x_{1}(t)=Y(t)-Y^{*}, \\
& x_{2}(t)=K(t)-K^{*}, \\
& x_{3}(t)=M(t)-M^{*} .
\end{aligned}
$$

The equilibrium point $E\left(Y^{*}, K^{*}, M^{*}\right)$ of system (6) can be translated into the original point, and the system is transformed as

$$
\begin{aligned}
& \frac{d x_{1}(t)}{d t} \\
& =\alpha\left(\frac{\left(2 Y^{*} m+n\right) x_{1}-d_{1} x_{2}+m x_{1}^{2}+b x_{3}(t-\tau)}{2 x_{1}+2 Y_{0}}\right), \\
& \frac{d x_{2}(t)}{d t}=-n x_{1}+d_{1} x_{2}+b x_{3}, \\
& \frac{d x_{3}(t)}{d t}=\left(\beta \delta+2 Y^{*} \delta_{1}\right) x_{1}-\beta \beta_{2} m x_{3}+\delta_{1} x_{1}^{2} .
\end{aligned}
$$

Considering Taylor expansion of the right members from (10) until the third order, we derive that

$$
\dot{x}(t)=A(t)+B x(t-\tau)+f(x(t)),
$$

where $f(x(t))$ is the nonlinear part of system (10) and its expression is

$$
f(x(t))=\frac{1}{2} B(x, x)+\frac{1}{6} C(x, x, x)+o\left\|x^{4}\right\|,
$$

where $B(x, y)$ and $C(x, y, u)$ are the bilinear and trilinear functions about the variation $x, y, u$ shown as

$$
\begin{gathered}
B_{i}(x, y)=\left.\sum_{j, k=1}^{2} \frac{\partial^{2} F_{i}(\xi, 0)}{\partial \xi_{j} \partial \xi_{k}}\right|_{\xi=0} x_{j} y_{k}, \quad i=1,2, \\
C_{i}(x, y, u)=\left.\sum_{j, k=1}^{3} \frac{\partial^{3} F_{i}(\xi, 0)}{\partial \xi_{j} \partial \xi_{k} \partial \xi_{l}}\right|_{\xi=0} x_{j} y_{k} u_{l}, \quad i=1,2,3 .
\end{gathered}
$$

According to the expressions above, we denote that

$$
\begin{aligned}
& A=\frac{1}{2 Y_{0}^{2}} \\
& {\left[\begin{array}{ccc}
\alpha Y_{0}\left(2 Y_{0} m+n\right)-\alpha\left(Y_{0}^{2} m+2 a\right) & -2 Y_{0}^{3} \alpha d_{1} & 0 \\
2 Y_{0}^{2} n & -2 Y_{0}^{2} d_{1} & 2 Y_{0}^{2} b \\
4 Y_{0}^{3} \delta_{1}+2 Y_{0}^{2} \beta \delta & 0 & -2 Y_{0}^{2} \beta \beta_{2}
\end{array}\right],} \\
& B=\frac{1}{2 Y_{0}}\left[\begin{array}{ccc}
0 & 0 & \alpha b \\
0 & 0 & 0 \\
0 & 0 & 0
\end{array}\right] \text {, }
\end{aligned}
$$

$$
f(x(t), x(t-\tau))=\left(\begin{array}{c}
f_{1}(x(t), x(t-\tau)) \\
0 \\
3 \delta_{1} x_{1}^{2}
\end{array}\right),
$$

where

$$
\begin{aligned}
f(x(t), x(t-\tau)) \\
=\frac{3}{2}\left(-\frac{\alpha m}{Y_{0}^{2}}+\frac{\alpha\left(2 Y_{0} m+n\right)}{Y_{0}^{3}}\right) x_{1}^{3}(t) \\
\quad+\frac{\alpha b}{Y_{0}^{3}} x_{1}^{2}(t) x_{3}(t-\tau)-\frac{\alpha d_{1}}{Y_{0}^{3}} x_{1}^{2}(t) x_{2}(t) \\
\quad+\frac{3}{2}\left(\frac{\alpha m}{Y_{0}}-\frac{\alpha\left(2 Y_{0} m+n\right)}{Y_{0}^{2}}\right) x_{1}^{2}(t) \\
\quad-\frac{\alpha b}{Y_{0}^{2}} x_{1}(t) x_{3}(t-\tau)+\frac{\alpha d_{1}}{Y_{0}^{2}} x_{1}(t) x_{2}(t) .
\end{aligned}
$$

Furthermore, the characteristic equation of the linear part of system (10) is

$$
\lambda^{3}+A \lambda^{2}+B \lambda+C+(D \lambda+E) e^{-\lambda \tau}=0,
$$

where

$$
\begin{aligned}
A= & -\frac{\alpha m}{2}+\beta \beta_{2}+d_{1}-\frac{\alpha n}{2 Y_{0}}+\frac{a \alpha}{Y_{0}^{2}}, \\
B= & -\frac{\alpha \beta m \beta_{2}}{2}-\frac{1}{2} \alpha m d_{1}+\beta \beta_{2} d_{1}-\frac{\alpha \beta n \beta_{2}}{2 Y_{0}}+\frac{a \alpha \beta \beta_{2}}{Y_{0}^{2}} \\
& +\frac{a \alpha d_{1}}{Y_{0}^{2}}, \\
C= & -\frac{\alpha \beta m \beta_{2} d}{2}+\alpha b d_{1} \delta_{1}+\frac{\alpha b \beta \delta d_{1}}{2 Y_{0}}+\frac{a \alpha \beta \beta_{2} d_{1}}{Y_{0}^{2}}, \\
D= & -\alpha b \delta_{1}-\frac{\alpha b \beta \delta}{2 Y_{0}}, \\
E= & -\alpha b d_{1} \delta_{1}-\frac{\alpha b \beta \delta d_{1}}{2 Y_{0}} .
\end{aligned}
$$


When $\tau=0$, according to the Routh-Hurwitz criteria, if the system meets

$\left(H_{1}\right)$

$$
\begin{aligned}
A & >0, \\
A(B+D)-(C+E) & >0,
\end{aligned}
$$

then all roots of (16) are negative, and at this time the equilibrium $E\left(Y^{*}, K^{*}, M^{*}\right)$ is stable; when $\tau \neq 0$, according to the Hopf bifurcating condition, we assume that (16) has a pair of conjugate pure imaginary characteristic roots, and let $\lambda=i \omega$, and substitute it into (16); we get

$$
-i \omega^{3}-A \omega^{2}+i B \omega+C+(i D \omega+E) e^{-i \tau \omega}=0 .
$$

Separate the real and imaginary parts of (19), and we get

$$
\begin{aligned}
& -A \omega^{2}+C+E \cos (\omega \tau)+D \omega \sin (\omega \tau)=0, \\
& -\omega^{3}+B \omega+D \omega \cos (\omega \tau)-E \sin (\omega \tau)=0 ;
\end{aligned}
$$

then

$$
\begin{aligned}
& -A \omega^{2}+C=-E \cos (\omega \tau)-\omega D \sin (\omega \tau), \\
& -\omega^{3}+B \omega=-\omega D \cos (\omega \tau)+E \sin (\omega \tau) .
\end{aligned}
$$

Square both sides of (21) and add them all:

$$
\begin{aligned}
\omega^{6} & +\left(A^{2}-2 B\right) \omega^{4}+\left(B^{2}-D^{2}-2 A C\right) \omega^{2}+C^{2}-E^{2} \\
& =0 .
\end{aligned}
$$

For simplicity, we denote that

$$
\omega^{6}+p \omega^{4}+q \omega^{2}+r=0,
$$

where $p=A^{2}-2 B, q=B^{2}-D^{2}-2 A C$, and $r=C^{2}-E^{2}$.

Let $z=\omega^{2}$; we obtain that

$$
h(z)=z^{3}+p z^{2}+q z+r=0 ;
$$

then, we discuss the roots of (24).

$\left(\mathrm{H}_{2}\right)$ When $r \geqslant 0$ and $p^{2}-3 q^{2}<0,(24)$ has no positive roots.

$\left(H_{3}\right)$ When $p^{2} q^{2}+18 p q r-27 r^{2}-4 p^{3} r-4 q^{3}<0$, (24) has a positive root and a pair of conjugate pure imaginary roots; that is, (24) has a positive root.

$\left(H_{4}\right)$ When $p^{2}-3 q=0, p q-9 r=0$, and $p<0$, (24) has three equal positive roots: $z_{k}=-p / 3, k=1,2,3$, and $\omega^{2}=$ $-p / 3$. That is, (24) has a unique positive root: $\omega=\sqrt{-p / 3}$.

$\left(H_{5}\right)$ When $p<(p q-9 r) /\left(p^{2}-3 q\right)<0$ and $p^{2} q^{2}-$ $18 p q r+27 r^{2}+4 p^{3} r+4 q^{3}=0$, (24) has positive roots. That is, $z_{1}=-p+(p q-9 r) /\left(p^{2}-3 q\right)$ and $z_{2}=z_{3}=$ $-(1 / 2)\left((p q-9 r) /\left(p^{2}-3 q\right)\right)$. And (24) has two solutions: $\omega_{1,2}= \pm \sqrt{-p+(p q-9 r) /\left(p^{2}-3 q\right)}$.

$\left(H_{6}\right)$ When $p^{2} q^{2}+18 p q r-27 r^{2}-4 p^{3} r-4 q^{3}>0$, (24) has three positive roots; that is, $z_{1}=\left(-p-2 \sqrt{p^{2}-3 q} \cos (\theta / 3)\right) / 3$ and $z_{2,3}=\left(-p-2 \sqrt{p^{2}-3 q} \sin (\theta / 3 \pm \pi / 6)\right) / 3$, where $\theta=$ $\arccos T, T=\left(2\left(p^{2}-3 q\right) p-3(p q-9 r)\right) / 2 \sqrt{\left(p^{2}-3 q\right)^{3}}$, and $\left(p^{2}-3 q>0,-1<T<1\right)$.

With regard to any $\omega_{j}$ above, we denote

$$
\tau_{j}^{k}=\frac{1}{\omega_{j}}\left[\theta_{j}+2 k \pi\right], \quad j=1,2,3, k=0,1,2, \ldots .
$$

Thus, according to (21),

$$
\begin{aligned}
\cos \left(\theta_{j}\right) & =\frac{D \omega_{j}^{4}+A E \omega_{j}^{2}-B D \omega_{j}^{2}-C E}{D^{2} \omega_{j}^{2}+E^{2}}, \\
\sin \left(\theta_{j}\right) & =\frac{\omega_{j}\left(A D \omega_{j}^{2}-E \omega_{j}^{2}+B E-C D\right)}{D^{2} \omega_{j}^{2}+E^{2}}
\end{aligned}
$$

and we will discuss transversality condition; we have the following lemmas.

Lemma 1. Assume that (16) has roots which is shown as $\lambda(\tau)=$ $\alpha(\tau)+i \omega(\tau)$, where $\alpha(\tau)$ and $\omega(\tau)$ meet the conditions $\alpha\left(\tau_{j}^{k}\right)=$ $0, \omega\left(\tau_{j}^{k}\right)=\omega_{j}$; then $\operatorname{sign}\left(d \operatorname{Re} \lambda\left(\tau_{j}^{k}\right) / d \tau_{j}^{k}\right)=\operatorname{sign}\left(h^{\prime}\left(\tau_{j}^{k}\right)\right)$.

Proof. Substitute $\lambda(\tau)=\alpha(\tau)+i \omega(\tau)$ into the characteristic equation (16), and differentiate it with respect to $\tau$; we have

$$
\begin{aligned}
3 \lambda^{2} \frac{d \lambda}{d \tau} & +2 A \lambda \frac{d \lambda}{d \tau}+B \frac{d \lambda}{d \tau}+D \frac{d \lambda}{d \tau} e^{-\lambda \tau} \\
& +(D \lambda+E) e^{-\lambda \tau}\left(-\frac{d \lambda}{d \tau} \tau-\lambda\right)=0
\end{aligned}
$$

and then

$$
\frac{d \lambda}{d \tau}=\frac{\left(D \lambda^{2}+E \lambda\right) e^{-\lambda \tau}}{3 \lambda^{2}+2 A \lambda+B+(D-D \lambda \tau-E \tau) e^{-\lambda \tau}}
$$

with respect to (16). Consider

$$
\left(D \lambda^{2}+E \lambda\right) e^{-\lambda \tau}=-\left(\lambda^{4}+A \lambda^{3}+B \lambda^{2}+C \lambda\right)
$$

substitute it into the equation above; we have

$$
\begin{aligned}
\operatorname{Re} & \frac{d \lambda\left(\tau_{j}^{k}\right)}{d \tau_{j}^{k}} \\
= & \frac{\omega_{j}^{2}}{S}\left[3 \omega_{j}^{4}+2 \omega_{j}^{2}\left(A^{2}-2 B\right)+B^{2}-2 A C-D^{2}\right] \\
= & \frac{\omega_{j}^{2}}{S}\left[3 \omega_{j}^{4}+2 \omega_{j}^{2} p+q\right]=\frac{\omega_{j}^{2} h^{\prime}\left(z_{j}\right)}{S},
\end{aligned}
$$

where

$$
\begin{aligned}
S= & {\left[-3 \omega_{j}^{2}+B-D E \tau_{j}^{k} \sin \omega_{j} \tau_{j}^{k}\right.} \\
& \left.+\left(D-E \tau_{j}^{k}\right) \cos \omega_{j} \tau_{j}^{k}\right]^{2}+\left[2 A \omega_{j}\right. \\
& \left.-\left(D-E \tau_{j}^{k}\right) \sin \omega_{j} \tau_{j}^{k}-D E \tau_{j}^{k} \cos \omega_{j} \tau_{j}^{k}\right]^{2}>0 .
\end{aligned}
$$


Thus, we have

$$
\operatorname{sign}\left(\frac{d \operatorname{Re} \lambda\left(\tau_{j}^{k}\right)}{d \tau_{j}^{k}}\right)=\operatorname{sign}\left(h^{\prime}\left(\tau_{j}^{k}\right)\right) .
$$

According to Lemma 1, we know that if system (10) meets hypothesis $\left(H_{3}\right)$ or $\left(H_{4}\right)$, then $\alpha^{\prime}\left(\tau^{k}\right)>0$; and if system (10) meets hypothesis $\left(H_{5}\right)$, then $\alpha^{\prime}\left(\tau_{1}^{k}\right)>0$ and $\alpha^{\prime}\left(\tau_{2}^{k}\right)=$ 0 ; if system (10) meets hypothesis $\left(H_{6}\right)$, then $\alpha^{\prime}\left(\tau_{1}{ }^{k}\right)>0$, $\alpha^{\prime}\left(\tau_{2}{ }^{k}\right)<0$, and $\alpha^{\prime}\left(\tau_{3}^{k}\right)>0$, where $\tau_{j}^{k}, j=1,2,3 ; k=$ $0,1,2, \ldots$, defined by $(25)$.

With regard to Lemma 1 we can conclude the following.

Lemma 2. With respect to (6),

(1) if system (6) meets $\left(H_{1}\right)$ and $\left(H_{2}\right)$, for any $\tau \in[0,+\infty)$, then the real parts of the roots of (16) are negative,

(2) if system (6) meets $\left(H_{1}\right)$ and $\left(H_{3}\right)$ or $\left(H_{1}\right)$ and $\left(H_{4}\right)$, then there exists a series of $\left\{\tau_{k}\right\}_{k \geq 0}$; when $\tau \in\left[0, \tau_{0}\right)$, all roots of (16) have negative part; and when $\tau=\tau_{k}$, (16) has a pair of conjugate pure imaginary roots; and when $\tau \in\left(\tau_{k}, \tau_{k+1}\right]$, (16) has $k+1$ pairs of characteristic roots with positive real part,

(3) if system (6) meets conditions $\left(H_{1}\right)$ and $\left(H_{5}\right)$, then there exists a series of $\left\{\tau_{1}^{k}\right\}_{k \geq 0}$; when all of characteristic roots are negative and when $\tau=\tau_{1}^{k}$, (16) has a pair of conjugate pure imaginary roots $\pm i \omega_{0}$,

(4) if system (6) meets conditions $\left(H_{1}\right)$ and $\left(H_{6}\right)$, there exists a series of $\left\{\tau_{j}^{k}\right\}_{k \geq 0}, k=0,1,2, \ldots, j=1,2,3$, and if $\tau_{1}^{0}<\tau_{2}^{0}<\tau_{3}^{0}$, then when $\tau \in\left[0, \tau_{1}^{0}\right)$, all the characteristic roots of (16) have negative real part; when $\tau \in\left(\tau_{1}^{0}, \tau_{2}^{0}\right]$, (16) has a pair of characteristic roots with real part.

Theorem 3. If system (6) meets hypothesis $\left(H_{1}\right)$, one can conclude the following:

(1) If system (6) meets hypothesis $\left(\mathrm{H}_{2}\right)$, for all $\tau \in$ $[0,+\infty)$, the system is stable near the equilibrium $E^{*}\left(Y^{*}, K^{*}, M^{*}\right)$.

(2) If system (6) meets hypothesis $\left(H_{3}\right)$ or $\left(H_{4}\right)$, for all $\tau \in\left[0, \tau_{0}\right)$, the system is stable near the equilibrium $E^{*}\left(Y^{*}, K^{*}, M^{*}\right)$; and when $\tau=\tau_{0}$, system (6) undergoes Hopf bifurcation at $E^{*}\left(Y^{*}, K^{*}, M^{*}\right)$.

(3) If system (6) meets hypothesis $\left(H_{5}\right)$ or $\left(H_{6}\right)$, one denotes $\tau_{0}=\min _{1 \leqslant j \leqslant 3}\left\{\tau_{j}^{0}\right\}$; system (6) is stable near the equilibrium $E^{*}\left(Y^{*}, K^{*}, M^{*}\right)$ for all $\tau \in\left[0, \tau_{0}\right)$, and when $\tau=\tau_{0}$, system (6) undergoes Hopf bifurcation at $E^{*}\left(Y^{*}, K^{*}, M^{*}\right)$; that is, system (6) has a branch of periodic solutions bifurcation the zero solution near $\tau=\tau_{0}$.

\section{Stability of Bifurcating Periodic Solutions}

In this section, formulation for determining the direction of Hopf bifurcation periodic solutions of system (6) at $\tau_{0}$ will be presented following the normal form method and center manifold theorem introduced by Hassard et al. in [17].

Denote $\tau$ as the criteria value of any $\tau=\tau_{k}(k=$ $1,2,3, \ldots)$ and assume that the pure imaginary characteristic roots of system (6) are $\pm i \omega$ with respect to $\tau$. For convenience, through linear transformation $t \rightarrow t / \tau$, we normalize the time delay based on system (10), and we get

$$
\begin{aligned}
& \frac{d X_{1}(t)}{d t} \\
& =\alpha \tau\left(\frac{\left(2 Y^{*} m+n\right) X_{1}-d_{1} X_{2}+m X_{1}^{2}+b X_{3}(t-1)}{2 x_{1}+2 Y_{0}}\right), \\
& \frac{d X_{2}(t)}{d t}=\tau\left[-n X_{1}+d_{1} X_{2}+b X_{3}\right] \\
& \frac{d X_{3}(t)}{d t}=\tau\left[\left(\beta \delta+2 Y^{*} \delta_{1}\right) X_{1}-\beta \beta_{2} m X_{3}+\delta_{1} X_{1}^{2}\right] .
\end{aligned}
$$

Assume that $\tau=\tau_{0}+\mu$; when $\mu=0$, the system undergoes Hopf bifurcation, and (33) can be transformed to the equation on $C\left([-1,0], \mathbf{R}^{3}\right)$; that is,

$$
\dot{Z}(t)=L(\mu) Z_{t}+f\left(Z_{t}, \mu\right) .
$$

For $\varphi=\left(\varphi_{1}, \varphi_{2}, \varphi_{3}\right)^{T} \in C$, we obtain

$$
\begin{aligned}
& L(\mu) \varphi=\left(\tau_{0}+\mu\right) \\
& \cdot\left(\begin{array}{c}
\alpha\left(\frac{m}{2}+\frac{1}{2} \frac{n}{Y_{0}}\right) \varphi_{1}(0)-\alpha d_{1} \varphi_{2}(0)+\alpha b \varphi(-1) \\
\frac{1}{2} \frac{n}{Y_{0}} \varphi_{1}(0)-d_{1} \varphi_{2}(0)+b \varphi_{3}(0) \\
\left(\frac{d_{1}}{2}+\frac{1}{2} \frac{\beta \delta}{Y_{0}}\right) \varphi_{1}(0)-\beta \beta_{2} \varphi_{3}(0)
\end{array}\right), \\
& f(\varphi, \mu)=\left(\begin{array}{c}
f_{1}(\varphi, \mu) \\
0 \\
3 \delta \varphi_{1}^{2}(0)
\end{array}\right),
\end{aligned}
$$

where

$$
\begin{aligned}
f(x(t), x(t-\tau)) & \\
= & \frac{3}{2}\left(-\frac{\alpha m}{Y_{0}^{2}}+\frac{\alpha\left(2 Y_{0} m+n\right)}{Y_{0}^{3}}\right) \varphi_{1}^{3}(0) \\
& +\frac{\alpha b}{Y_{0}^{3}} \varphi_{1}^{2}(0) \varphi_{3}(-1)-\frac{\alpha d_{1}}{Y_{0}^{3}} \varphi_{1}^{2}(0) \varphi_{2}(0) \\
& +\frac{3}{2}\left(\frac{\alpha m}{Y_{0}}-\frac{\alpha\left(2 Y_{0} m+n\right)}{Y_{0}^{2}}\right) \varphi_{1}^{2}(0) \\
& -\frac{\alpha b}{Y_{0}^{2}} \varphi_{1}(0) \varphi_{3}(-1)+\frac{\alpha d_{1}}{Y_{0}^{2}} \varphi_{1}(0) \varphi_{2}(0),
\end{aligned}
$$

and we easily know that the characteristic equation of $L$ is

$$
\operatorname{det}\left(\lambda I-\int_{-1}^{0} e^{\lambda \theta} d \theta \eta(\mu, \theta)\right)=0 .
$$


According to Riesz theorem, we take the function of bounded variation; that is, $\eta(\theta)=\tau A \delta(\theta)+\tau B \delta(\theta+1)$. Consider

$$
L(\mu) \varphi=\int_{-1}^{0} e^{\lambda \theta} d \eta(\theta) \varphi(\theta), \quad \varphi \in C
$$

Then, we can define infinitely small generating element $A(\mu)=A$ with respect to (34); we have

$$
A \varphi(\theta)= \begin{cases}\frac{d \varphi(\theta)}{d \theta} ; & \theta \in[-1,0) \\ L(\mu) \varphi=\int_{-1}^{0} d \eta(\theta) \varphi(\theta) ; & \theta=0\end{cases}
$$

$$
\left(\begin{array}{ccc}
i \omega_{0} \tau_{0}-\alpha\left(m+\frac{n}{2 Y_{0}}\right) & Y_{0} \alpha d_{1} & \alpha b e^{-i \omega_{0} \tau_{0}} \\
-\frac{n}{2 Y_{0}} & i \omega_{0} \tau_{0}+d_{1} & -b \\
-d_{1}-\frac{\beta \delta}{2 Y_{0}} & 0 & i \omega_{0} \tau_{0}+\beta \beta_{2}
\end{array}\right)\left(\begin{array}{l}
p_{1}(0) \\
p_{2}(0) \\
p_{3}(0)
\end{array}\right)=0
$$

where

$$
\begin{aligned}
& p_{1}(0)=1, \\
& p_{2}(0)=\frac{i \omega_{0} \tau_{0} n+2 Y_{0} b d_{1}+b \beta \delta+n \beta \beta_{2}}{2 Y_{0}\left(-\omega_{0}^{2} \tau_{0}^{2}+i \omega_{0} \tau_{0} \beta \beta_{2}+i d_{1} \omega_{0} \tau_{0}+d_{1} \beta \beta_{2}\right)}, \\
& p_{3}(0)=\frac{2 i Y_{0} \tau_{0} \omega_{0} d_{1}+i \beta \delta \tau_{0} \omega_{0}+2 d_{1}^{2} Y_{0}+d_{1} \beta \delta}{2 Y_{0}\left(-\omega_{0}^{2} \tau_{0}^{2}+i \omega_{0} \tau_{0} \beta \beta_{2}+i \omega_{0} \tau_{0} d_{1}+\beta \beta_{2} d_{1}\right)},
\end{aligned}
$$

$$
\left(\begin{array}{ccc}
-i \omega_{0} \tau_{0}-\alpha\left(m+\frac{n}{2 Y_{0}}\right) & -\frac{n}{2 Y_{0}} & -d_{1}-\frac{\beta \delta}{2 Y_{0}} \\
\alpha d_{1} & -i \omega_{0} \tau_{0}+d_{1} & 0 \\
-\alpha b e^{i \omega_{0} \tau_{0}} & -b & -i \omega_{0} \tau_{0}+\beta \beta_{2}
\end{array}\right)\left(\begin{array}{l}
q_{1}(0) \\
q_{2}(0) \\
q_{3}(0)
\end{array}\right)=0 .
$$

By calculating, $q_{1}(0)=1, q_{2}(0)=\alpha d_{1} /\left(i \omega_{0} \tau_{0}-d_{1}\right), q_{3}(0)=$ $-\left(\alpha b\left(\left(i \omega_{0} \tau_{0}-d_{1}\right) e^{i \omega_{0} \tau_{0}}+d_{1}\right) /\left(i \omega_{0} \tau_{0}-d_{1}\right)\left(i \omega_{0} \tau_{0}-\beta \beta_{2}\right)\right)$; thus, $q(s)=D\left(q_{1}(0), q_{2}(0), q_{3}(0)\right)^{T} e^{i \omega_{0} \tau_{0}}$ according to bilinear form $\langle q, p\rangle=\bar{q}(0) p(0)-\int_{-1}^{0} \int_{0}^{\theta} \bar{q}(\xi-\theta) d \eta(\theta) p(\xi) d \xi$; that is,

$$
\begin{aligned}
& \langle q(s), p(\theta)\rangle=\bar{D}\left(1, \bar{q}_{1}(0), \bar{q}_{2}(0)\right) \\
& \quad \cdot\left(1, p_{1}(0), p_{2}(0)\right)^{T} \\
& \quad-\int_{-1}^{0} \int_{0}^{\theta}\left(\bar{q}_{1}(0), \bar{q}_{2}(0), \bar{q}_{3}(0)\right) e^{-i \omega_{0} \tau_{0}(\xi-\theta)} d \eta(\theta)
\end{aligned}
$$

$\forall \psi \in C\left([0,1], C^{3}\right)$; denote $A^{*}$ as the formally adjoint operator of $A$ :

$$
A^{*} \psi(s)= \begin{cases}-\frac{d \psi(s)}{d s}, & s \in(0,1] \\ \int_{-1}^{0} \psi(-s) d \eta(s), & s=0\end{cases}
$$

and assume that $q(s)=D q(0) e^{i \omega_{0} \tau_{0} s}$ is the characteristic vector with respect to the characteristic value $-i \omega_{0} \tau_{0}$ of $A^{*}$, where $q(0)=\left(q_{1}(0), q_{2}(0), q_{3}(0)\right)^{T}$ meets the characteristic equation $D\left(-i \omega_{0} \tau_{0} I-A^{T}-B^{T} e^{i \omega_{0} \tau_{0}}\right) q(0)=0$. Consider

$$
\begin{aligned}
& \cdot\left(p_{1}(0), p_{2}(0), p_{3}(0)\right) e^{i \omega_{0} \tau_{0} \xi}=\bar{D}[1 \\
& +\bar{q}_{1}(0) p_{1}(0)+\bar{q}_{2}(0) p_{2}(0) \\
& \left.-\alpha b q_{1}(0) p_{3}(0) e^{-i \omega_{0} \tau_{0}}\right]
\end{aligned}
$$

where $\bar{D}^{-1}=\bar{q}_{1}(0) p_{1}(0)+\bar{q}_{2}(0) p_{2}(0)+\bar{q}_{3}(0) p_{3}(0)-$ $\alpha b \bar{q}_{1}(0) p_{3}(0) e^{-\mathrm{i} \omega_{0} \tau_{0}}$. 
According to the solution of (33) at $\tau=\tau_{0}$, we define $z(t)=\left\langle q, x_{t}\right\rangle$ and denote

$$
W(t, \theta)=x_{t}(\theta)-z(t) p(\theta)-\bar{z}(t) \bar{p}(\theta) ;
$$

thus, on center manifold $W(t, \theta)=W(z, \bar{z}, \theta)$, where $W(z, \bar{z}, \theta)$ can be expressed into power series form with variations $z$ and $\bar{z}$,

$$
\begin{aligned}
W(z, \bar{z}, \theta)= & W_{20}(\theta) \frac{z^{2}}{2}+W_{11}(\theta) z \bar{z}+W_{02}(\theta) \frac{\bar{z}^{2}}{2} \\
& +\cdots .
\end{aligned}
$$

We can obtain the form of (34) on center manifold which is shown as follows:

$$
\begin{aligned}
\dot{z}(t)= & i \omega_{0} \tau_{0} z(t) \\
& +\bar{q}(0) f\left(\tau_{0}, z p(\theta)+\bar{z} \bar{p}(\theta)+W(z, \bar{z}, \theta)\right) \\
\triangleq & i \omega_{0} \tau_{0} z(t)+\bar{q}(0) f_{0}(z, \bar{z}),
\end{aligned}
$$

and we denote

$$
f_{0}(z, \bar{z})=f_{z^{2}} \frac{z^{2}}{2}+f_{z \bar{z}} z \bar{z}+f_{\bar{z}^{2}} \frac{\bar{z}^{2}}{2}+f_{z^{2} \bar{z}} \frac{z^{2} \bar{z}}{2}+\cdots ;
$$

for simplicity, we denote (49) as the form shown as

$$
\dot{z}(t)=I \omega_{0} \tau_{0} z(t)+g(z, \bar{z})
$$

and denote

$$
g(z, \bar{z})=g_{20} \frac{z^{2}}{2}+g_{11} z \bar{z}+g_{02} \frac{\bar{z}^{2}}{2}+g_{21} \frac{z^{2} \bar{z}}{2}+\cdots,
$$

where (51) is the norm form of (34) on center manifold.

In order to obtain the direction and stability of Hopf bifurcation, we need to calculate regulating coefficients $g_{20}$, $g_{11}, g_{02}$, and $g_{21}$ and compare with formulas (48), (50), and (51); we have

$$
\begin{aligned}
& g_{20}=\bar{q}(0) f_{z^{2}}, \\
& g_{11}=\bar{q}(0) f_{z \bar{z}}, \\
& g_{02}=\bar{q}(0) f_{\bar{z}^{2}}, \\
& g_{21}=\bar{q}(0) f_{z^{2} \bar{z}} .
\end{aligned}
$$

With regard to (47),

$$
\begin{aligned}
x_{t}(\theta)= & z(t) p(\theta)+\bar{z}(t) \bar{p}(\theta)+W(z, \bar{z}, \theta) \\
= & \left(\begin{array}{l}
p_{1}(0) \\
p_{2}(0) \\
p_{3}(0)
\end{array}\right) e^{i \omega_{0} \tau_{0} \theta} z(t) \\
& +\left(\begin{array}{c}
\bar{p}_{1}(0) \\
\bar{p}_{2}(0) \\
\bar{p}_{3}(0)
\end{array}\right) e^{i \omega_{0} \tau_{0} \theta} \bar{z}(t)+\left(\begin{array}{c}
W_{20}^{1}(\theta) \\
W_{20}^{2}(\theta) \\
W_{20}^{3}(\theta)
\end{array}\right) \frac{z^{2}}{2} \\
& +\left(\begin{array}{c}
W_{11}^{1}(\theta) \\
W_{11}^{2}(\theta) \\
W_{11}^{3}(\theta)
\end{array}\right) z \bar{z}+\left(\begin{array}{c}
W_{02}^{1}(\theta) \\
W_{02}^{2}(\theta) \\
W_{02}^{3}(\theta)
\end{array}\right) \frac{\bar{z}^{2}}{2}+\cdots
\end{aligned}
$$

Furthermore, we obtain that

$$
\begin{aligned}
x(t)= & p(0) z(t)+\bar{p}(0) \bar{z}(t)+W_{20}(0) \frac{z^{2}}{2} \\
& +W_{11}(0) z \bar{z}+W_{02}(0) \frac{\bar{z}^{2}}{2}+\cdots, \\
x(t-1)= & p(0) z(t) e^{-i \omega_{0} \tau_{0}}+\bar{p}(0) \bar{z}(t) e^{i \omega_{0} \tau_{0}} \\
& +W_{20}(-1) \frac{z^{2}}{2}+W_{11}(-1) z \bar{z} \\
& +W_{02}(-1) \frac{\bar{z}^{2}}{2}+\cdots .
\end{aligned}
$$

Substitute $x(t)$ into formula $f_{0}$ and compare the coefficient with formula (50); and

$$
\begin{aligned}
f_{z^{2}} & =\left(f_{z^{2}}^{1}, 0,3 \delta_{1} \bar{p}_{1}^{2}(0)\right)^{T} \\
f_{z \bar{z}} & =\left(f_{z \bar{z}}^{1}, 0,6 \delta_{1} p_{1}(0) \bar{p}_{1}(0)\right)^{T}, \\
f_{\bar{z}^{2}} & =\left(f_{\bar{z}^{2}}^{1}, 0,3 \delta_{1} \bar{p}_{1}^{2}(0)\right)^{T}, \\
f_{z^{2} \bar{z}} & =\tau_{0}\left(f_{z^{2} \bar{z}}^{1}, 0,6 \delta_{1} p_{1}(0) W_{11}^{1}(0)\right. \\
& \left.+3 \delta_{1} \bar{p}_{1}(0) W_{20}^{1}(0)\right)^{T},
\end{aligned}
$$

where

$$
\begin{aligned}
& f_{z^{2}}^{1}=\frac{\alpha d_{1}}{S^{2}} p_{1}(0) p_{2}(0)-\frac{\alpha b}{S^{2}} p_{1}(0) p_{3}(0) \\
& -\frac{3 \alpha n}{2 S^{2}} p_{1}^{2}(0)+3 \frac{\alpha a p_{1}^{2}(0)}{S^{3}}, \\
& f_{z \bar{z}}^{1}=\frac{\alpha d_{1}}{S^{2}} p_{1}(0) \bar{p}_{2}(0)+\frac{\alpha d_{1}}{S^{2}} \bar{p}_{1}(0) p_{2}(0) \\
& +\frac{6 a \alpha}{S^{3}} p_{1}(0) \bar{p}_{1}(0)-\frac{3 \alpha n}{S^{2}} p_{1}(0) \bar{p}_{1}(0) \\
& -\frac{\alpha b}{S^{2}} p_{1}(0) \bar{p}_{3}(0)-\frac{\alpha b}{S^{2}} \bar{p}_{1}(0) p_{3}(0), \\
& f_{\bar{z}^{2}}^{1}=\frac{\alpha d_{1} \bar{p}_{1}(0) \bar{p}_{2}(0)}{S^{2}}-\frac{\alpha b \bar{p}_{1}(0) \bar{p}_{3}^{2}(0)}{S^{2}} \\
& -\frac{3 \alpha n \bar{p}_{1}^{2}(0)}{2 S^{2}}+\frac{3 \alpha a \bar{p}_{1}^{2}(0)}{S^{3}} \\
& f_{z^{2} \bar{z}}^{1}=-2 \frac{\alpha d_{1} p_{1}(0) \bar{p}_{1}(0) p_{2}(0)}{S^{3}} \\
& +2 \frac{\alpha b p_{1}(0) \bar{p}_{1}(0) p_{3}(0)}{S^{3}} \\
& +3 \frac{\alpha a \bar{p}_{1}(0) W_{20}^{1}(0)}{S^{3}}-9 \frac{\alpha a p_{1}^{2}(0) \bar{p}_{1}(0)}{S^{4}} \\
& -\frac{\alpha d_{1} p_{1}^{2}(0) \bar{p}_{2}(0)}{S^{3}}-\frac{3 \alpha n \bar{p}_{1}(0) W_{20}^{1}(0)}{2 S^{2}}
\end{aligned}
$$




$$
\begin{aligned}
& +\frac{\alpha d_{1} \bar{p}_{1}(0) W_{20}^{2}(0)}{2 S^{2}}+\frac{\alpha d_{1} W_{20}^{1}(0) \bar{p}_{2}(0)}{2 S^{2}} \\
& +\frac{9 \alpha n p_{1}^{2}(0) \bar{p}_{1}(0)}{2 S^{3}}-\frac{\alpha b \bar{p}_{1}(0) W_{20}^{3}(0)}{2 S^{2}} \\
& +\frac{\alpha b p_{1}^{2}(0) \bar{p}_{3}(0)}{S^{3}}-\frac{\alpha b W_{20}^{1}(0) \bar{p}_{2}(0)}{2 S^{2}} \\
& +\frac{\alpha d_{1} W_{11}^{1}(0) p_{2}(0)}{S^{2}}-\frac{\alpha b p_{1}(0) W_{11}^{3}(-1)}{S^{2}} \\
& -\frac{\alpha b W_{11}^{1}(0) p_{3}(0)}{S^{2}}-3 \frac{\alpha n p_{1} W_{11}^{1}(0)}{S^{2}} \\
& +6 \frac{\alpha a p_{1} W_{11}^{1}(0)}{S^{3}}+\frac{\alpha d_{1} p_{1} W_{11}^{2}(0)}{S^{2}} .
\end{aligned}
$$

Substitute $f_{z^{2}}, f_{z \bar{z}}, f_{\bar{z}^{2}}$ into formula (53); then

$$
\begin{aligned}
& g_{20}=\bar{q}_{1}(0) f_{z^{2}}^{1}+3 \delta_{1} \bar{q}_{3}(0) \bar{p}_{1}^{2}(0), \\
& g_{11}=\bar{q}_{1}(0) f_{z \bar{z}}^{1}+6 \delta_{1} \bar{q}_{3}(0) p_{1}(0) \bar{p}_{1}(0), \\
& g_{02}=\bar{q}_{1}(0) f_{\bar{z}^{2}}^{1}+3 \delta_{1} \bar{q}_{3}(0) \bar{p}_{1}^{2}(0) .
\end{aligned}
$$

However, there are some unknown terms, $W_{20}(0)$, $W_{11}(0), W_{11}(-1)$, in formula $f_{z^{2} \bar{z}}$, and thus in order to obtain $g_{21}$ we must calculate $W_{20}(\theta), W_{11}(\theta)$ firstly, and with respect to formula (52) we know that $\dot{W}=\dot{x}_{t}-\dot{z} p-\bar{z} \bar{p}$; that is,

$$
\dot{W}= \begin{cases}A W-g p(\theta)-\bar{g} \bar{p}(\theta), & \theta \in[-1,0), \\ A W-g p(0)-\bar{g} \bar{p}(0)+f_{0}, & \theta=0 .\end{cases}
$$

On the other hand, the system can be transferred into $\dot{W}=W_{z} \dot{z}+W_{\bar{z}} \dot{\bar{z}}$, the center manifold near the origin point:

$$
\begin{aligned}
\dot{W}= & W_{z} \dot{z}+W_{\bar{z}} \dot{\bar{z}} \\
= & {\left[W_{20}(\theta) z+W_{11}(\theta) \bar{z}\right] \dot{z} } \\
& +\left[W_{11}(\theta) z+W_{20}(\theta) \bar{z}\right] \dot{\bar{z}} \\
= & {\left[W_{20}(\theta) z+W_{11}(\theta) \bar{z}\right]\left(i \omega_{0} \tau_{0} z+g(z, \bar{z})\right) } \\
& +\left[W_{11}(\theta) z+W_{02}(\theta) \bar{z}\right]\left(i \omega_{0} \tau_{0} z+\bar{g}(z, \bar{z})\right) .
\end{aligned}
$$

Compared with the coefficients of $z^{2} / 2$ and $z \bar{z}$ in formulas (59) and (60),

$$
\begin{aligned}
& \left(2 i \omega_{0} \tau_{0} I-A\right) W_{20}(\theta) \\
& \quad= \begin{cases}-g_{20} p(\theta)-\bar{g}_{02} \bar{p}(\theta), & \theta \in[-1,0), \\
-g_{20} p(0)-\bar{g}_{02} \bar{p}(0)+f_{z^{2}}, & \theta=0,\end{cases} \\
& -A W_{11}(\theta) \\
& = \begin{cases}-g_{11} p(\theta)-\bar{g}_{11} \bar{p}(\theta), & \theta \in[-1,0), \\
-g_{11} p(0)-\bar{g}_{11} \bar{p}(0)+f_{z \bar{z}}, & \theta=0 .\end{cases}
\end{aligned}
$$

According to formulas (37) and (61), we have

$$
W_{20}(\theta)=2 i \omega_{0} \tau_{0} W_{20}(\theta)+g_{20} p(\theta)+\bar{g}_{02} \bar{p}(\theta) .
$$

Substitute $p(\theta)=p(0) e^{i \omega_{0} \tau_{0} \theta}$ into formula above; then

$$
\begin{aligned}
W_{20}(\theta)= & \frac{i g_{20}}{\omega_{0} \tau_{0}} p(0) e^{i \omega_{0} \tau_{0} \theta}+\frac{i \bar{g}_{02}}{3 \omega_{0} \tau_{0}} \bar{p}(0) e^{-i \omega_{0} \tau_{0} \theta} \\
& +E_{1} e^{2 i \omega_{0} \tau_{0} \theta} .
\end{aligned}
$$

Furthermore, we have

$$
\begin{aligned}
\int_{-1}^{0} d \eta(\theta) W_{20}(\theta)= & 2 i \omega_{0} \tau_{0} W_{20}(0)+g_{20} p(0) \\
& +\bar{g}_{02} \bar{p}(0)-f_{z^{2}}
\end{aligned}
$$

Substitute formula (64) into (65) with respect to $\left(i \omega_{0} \tau_{0} I-\right.$ $\left.\int_{-1}^{0} e^{i \omega_{0} \tau_{0} \theta} d \eta(\theta)\right) p(0)=0$; we have

$$
\left[2 i \omega_{0} \tau_{0} I-\int_{-1}^{0} e^{2 i \omega_{0} \tau_{0} \theta} d \eta(\theta)\right] E_{1}=f_{z^{2}} .
$$

Denote

$$
\left[2 i \omega_{0} \tau_{0} I-\int_{-1}^{0} e^{2 i \omega_{0} \tau_{0} \theta} d \eta(\theta)\right]^{-1}=\left(\begin{array}{lll}
a_{11} & a_{12} & a_{13} \\
a_{21} & a_{22} & a_{23} \\
a_{31} & a_{32} & a_{33}
\end{array}\right)
$$

then,

$$
E_{1}=\frac{1}{S_{1}}\left(\begin{array}{c}
a_{11} f_{z^{2}}^{1}+3 a_{13} \delta_{1} \bar{p}_{1}^{2} \\
a_{21} f_{z^{2}}^{1}+3 a_{23} \delta_{1} \bar{p}_{1}^{2} \\
a_{31} f_{z^{2}}^{1}+3 a_{33} \delta_{1} \bar{p}_{1}^{2}
\end{array}\right)=\left(\begin{array}{c}
E_{1}^{1} \\
E_{1}^{2} \\
E_{1}^{3}
\end{array}\right),
$$

where

$$
\begin{aligned}
a_{11}= & -2\left(2 i \omega_{0}+d_{1}\right)\left(2 i \omega_{0}+\beta \beta_{2}\right) Y_{0}, \\
a_{12}= & \alpha d_{1}\left(2 i \omega_{0}+\beta \beta_{2}\right), \\
a_{13}= & -\alpha b\left(4 i e^{-2 i \omega_{0} \tau_{0}} Y_{0} \omega_{0}+2 e^{-2 i \omega_{0} \tau_{0}} Y_{0} d_{1}-d_{1}\right), \\
a_{21}= & -2\left(2 i n \omega_{0}+2 Y_{0} b \delta_{1}+b \beta \delta+\beta n \beta_{2}\right) Y_{0}, \\
a_{22}= & 4 e^{-2 i \omega_{0} \tau_{0}} Y_{0}^{2} \alpha b \delta_{1}+2 e^{-2 i \omega_{0} \tau_{0}} Y_{0} \alpha b \beta \delta+8 Y_{0} \omega_{0}^{2} \\
& +4 i Y_{0} \alpha m \omega_{0}-4 i Y_{0} \beta \omega_{0} \beta_{2}+2 Y_{0} \alpha \beta m \beta_{2} \\
& +2 i \alpha n \omega_{0}+\alpha, \\
a_{23}= & -b\left(2 e^{-2 i \omega_{0} \tau_{0}} \alpha n Y_{0}+4 i Y_{0} \omega_{0}-2 Y_{0} \alpha m-\alpha n\right), \\
a_{31}= & -2\left(2 i \omega_{0}+d_{1}\right)\left(2 Y_{0} \delta_{1}+\beta \delta\right) Y_{0}, \\
a_{32}= & \alpha d_{1}\left(2 Y_{0} \delta_{1}+\beta \delta\right), \\
a_{33}= & 4 i Y_{0} \alpha m \omega_{0}-4 i Y_{0} \omega_{0} d_{1}+2 i \alpha n \omega_{0}+2 Y_{0} \alpha m d_{1} \\
& +8 Y_{0} \omega_{0}^{2},
\end{aligned}
$$




$$
\begin{aligned}
S_{1}= & 4 i Y_{0} \alpha m \omega_{0} d_{1}+4 i Y_{0} \alpha \beta m \omega_{0} \beta_{2} \\
& +4 e^{-2 i \omega_{0} \tau_{0}} Y_{0}^{2} \alpha b d_{1} \delta_{1}+2 e^{-2 i \omega_{0} \tau_{0}} Y_{0} \alpha b \beta \delta d_{1} \\
& +16 i Y_{0} \omega_{0}^{3}-8 Y_{0} \alpha m \omega_{0}^{2}+8 Y_{0} \beta \omega_{0}^{2} \beta_{2} \\
& +8 i e^{-2 i \omega_{0} \tau_{0}} Y_{0}^{2} \alpha b \omega_{0} \delta_{1}+8 Y_{0} \omega_{0}^{2} d_{1}-4 \alpha n \omega_{0}^{2} \\
& +4 i e^{-2 i \omega_{0} \tau_{0}} Y_{0} \alpha b \beta \delta \omega_{0}-4 i Y_{0} \beta \omega_{0} \beta_{2} d_{1} \\
& +2 i \alpha \beta n \omega_{0} \beta_{2}+2 Y_{0} \alpha \beta m \beta_{2} d_{1}-2 Y_{0} \alpha b d_{1} \delta_{1} \\
& -\alpha b \beta \delta d_{1} .
\end{aligned}
$$

In a similar way, with regard to formulas (37) and (62), we have $\dot{W}_{11}(\theta)=g_{11} p(\theta)+\bar{g}_{11} \bar{p}(\theta)$ and we get

$$
\begin{aligned}
W_{11}(\theta)= & -\frac{i g_{11}}{\omega_{0} \tau_{0}} p(0) e^{i \omega_{0} \tau_{0} \theta}+\frac{i \bar{g}_{11}}{\omega_{0} \tau_{0}} \bar{p}(0) e^{-i \omega_{0} \tau_{0} \theta} \\
& +E_{2} .
\end{aligned}
$$

According to (62), when $\theta=0$,

$$
\int_{-1}^{0} d \eta(0, \theta) W_{11}(\theta)=g_{11} p(0)+\bar{g}_{11} \bar{p}(0)-f_{z \bar{z}}
$$

Substitute (70) into (71) and we have $-\int_{-1}^{0} d \eta(\theta) E_{2}=f_{z \bar{z}}$, where

$$
\begin{aligned}
& E_{2}=\frac{1}{S_{2}}\left(\begin{array}{c}
-2 \frac{\beta \beta_{2} Y_{0}}{\alpha} f_{z \bar{z}}^{1}+6 b\left(1-2 Y_{0}\right) \delta_{1} p_{1}(0) \bar{p}_{1}(0) \\
-\frac{\left(4 Y_{0} b \delta_{1}+2 b \beta \delta+2 \beta n \beta_{2}\right) Y_{0}}{\alpha d_{1}} f_{z \bar{z}}^{1}+\frac{6 b\left(2 Y_{0} m-2 Y_{0} n+n\right) \delta_{1} p_{1}(0) \bar{p}_{1}(0)}{d_{1}} \\
-\frac{\left(4 Y_{0} \delta_{1}+2 \beta \delta\right) Y_{0}}{\alpha} f_{z \bar{z}}^{1}+12 Y_{0} m \delta_{1} p_{1}(0) \bar{p}_{1}(0)
\end{array}\right), \\
& S_{2}=4 Y_{0}^{2} b \delta_{1}+2 Y_{0} b \beta \delta+2 Y_{0} \beta m \beta_{2}-2 Y_{0} b \delta_{1}-b \beta \delta
\end{aligned}
$$

with regard to (64) and (70), we have

$$
\begin{aligned}
W_{20}(\theta)= & \frac{i g_{20}}{\omega_{0} \tau_{0}} p_{1}(0) e^{i \omega_{0} \theta}+\frac{i \bar{g}_{02}}{3 \omega_{0} \tau_{0}} \bar{p}_{1}(0) e^{i \omega_{0} \theta} \\
& +E_{1} e^{2 i \omega_{0} \theta} \\
W_{11}(\theta)= & -\frac{i g_{11}}{\omega_{0} \tau_{0}} p_{1}(0) e^{i \omega_{0} \theta}+\frac{i \bar{g}_{11}}{\omega_{0} \tau_{0}} \bar{p}_{1}(0) e^{i \omega_{0} \theta} \\
& +E_{2} e^{2 i \omega_{0} \theta} .
\end{aligned}
$$

And we acquired $g_{21}$ shown as follows from the formula above:

$$
\begin{aligned}
g_{21}= & \bar{q}_{1}(0) \tau_{0} f_{z^{2} \bar{z}}^{1}+6 \delta_{1} \bar{q}_{1}(0) p_{1}(0) W_{11}^{1}(0) \\
& +3 \delta_{1} \bar{q}_{1}(0) \bar{p}_{1}(0) W_{20}^{1}(0) .
\end{aligned}
$$

Now, we have acquired all values of $g_{20}, g_{11}, g_{02}, g_{21}$; thus we get normal form on center manifolds (51) of (34). From the certain equation, we can get the parameters of Hopf bifurcation such as $C_{1}(0), \mu_{2}$, and $\beta_{2}$ which are shown as

$$
\begin{aligned}
C_{1}(0) & =\frac{i}{2 \omega_{0}}\left(g_{20} g_{11}-2\left|g_{11}\right|^{2}-\frac{\left|g_{02}\right|^{2}}{3}\right)+\frac{g_{21}}{2}, \\
\mu_{2} & =-\frac{\operatorname{Re}\left\{C_{1}(0)\right\}}{\operatorname{Re} \lambda^{\prime}\left(\tau_{0}\right)}, \\
\beta_{2} & =2 \operatorname{Re}\left\{C_{1}(0)\right\}
\end{aligned}
$$

$$
\begin{gathered}
\tau_{2}=-\frac{\operatorname{Im}\left(C_{1}(0)\right)+\mu_{2} \omega^{\prime}(0)}{\omega_{0}}, \\
P=\frac{2 \pi}{\omega_{0}}\left[1+\tau_{2} \varepsilon^{2}+O\left(\varepsilon^{4}\right)\right] .
\end{gathered}
$$

From the parameters $\mu_{2}$ and $\beta_{2}$, the direction of Hopf bifurcation at $\tau=\tau_{0}$ and the stability of periodic solution generated by the Hopf bifurcation can be presented, where $\mu_{2}$ decides the direction of the Hopf bifurcation and the parameter $\beta_{2}$ gives the stability of the periodic solution which is shown as

$$
\begin{aligned}
& x(t, \mu(\varepsilon)) \\
& =2 \varepsilon \operatorname{Re}\left[q(0) e^{i \omega_{0} t}\right] \\
& +2 \varepsilon^{2} \operatorname{Re}\left[q(0)\left(\frac{g_{20}}{2 i \omega_{0}} e^{2 i \omega_{0} t}-\frac{g_{11}}{i \omega_{0}}-\frac{g_{02}}{6 i \omega_{0}} e^{2 i \omega_{0} t}\right)\right] \\
& +e^{2} \operatorname{Re}\left[W_{20}(0) e^{2 i \omega_{0} t}+W_{11}(0)\right]+O\left(\varepsilon^{3}\right), \\
& 0 \leqslant t \leqslant P(\varepsilon)=\frac{2 \pi}{\omega_{0}}\left[1+\tau_{2} \varepsilon^{2}+\cdots\right] .
\end{aligned}
$$

\section{Numerical Simulation}

In order to reflect the basic situation of the macroeconomic business cycle system with currency supply delay and confirm the theoretical analysis above, we do some numerical simulation to system (6). 


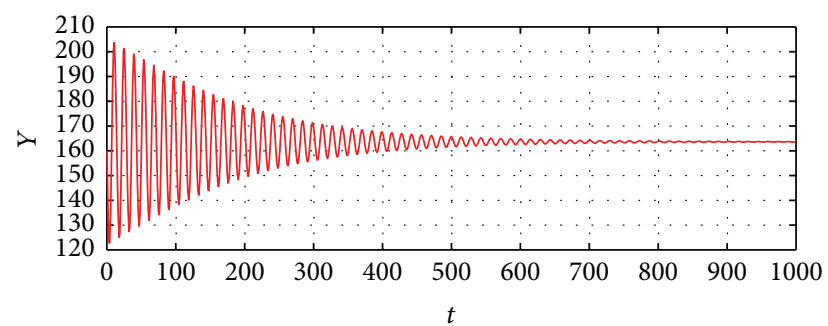

FIgURE 1: History of gross domestic product $Y$ when $\tau=0$.

We take parameters $\alpha, \beta, \beta_{2}, m, n, d_{1}, a, b, c, \delta, \delta_{1}, r_{0}$ with the value shown as follows:

$$
\begin{aligned}
\alpha & =1, \\
\beta & =10, \\
m & =-0.66, \\
n & =15, \\
d_{1} & =0.3, \\
a & =108, \\
b & =1, \\
\delta_{1} & =-0.3, \\
\delta & =10, \\
\beta_{2} & =1, \\
r_{0} & =3 .
\end{aligned}
$$

$$
\begin{aligned}
& \frac{d K(t)}{d t}=15 Y(t)-0.3 K(t)+M(t) \\
& \frac{d M(t)}{d t}=-0.3 Y^{2}(t)+100 Y(t)-10 M(t)-10 .
\end{aligned}
$$

Through calculating, the system has a unique equilibrium point $E^{*}(163.6363,1046.3,122)$, and we let $\tau=0$; according to Theorem 3 , when the system meets hypothesis $\left(H_{1}\right)$, the equilibrium is stable as shown in Figures 1-4.

We conclude that the gross domestic product $Y$ and physical capital stock $K$ and the currency supply $M$ undergo some oscillation at the initial time and then converge into the equilibrium point $E^{*}(163.6363,1046.3,122)$ rapidly.

Furthermore, we consider system (79); (24) has only one positive solution, $z_{0}^{+}=0.1615$. According to (25), we obtain $\tau^{k}=22.045+4.9762 k \pi$. Through the calculation by (75), we obtain that $C_{1}(0)=-2.7964+4.3725 i ; \mu_{2}>0 ; \beta_{2}=-5.5928<$ 0 , and according to Theorem 3 , Hopf bifurcation is generated

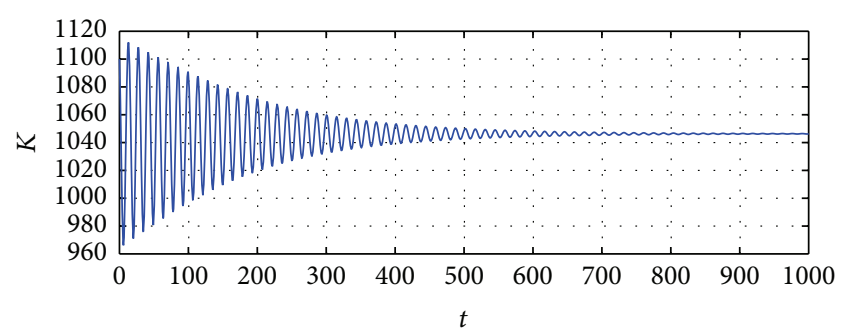

FIgURE 2: History of physical capital stock $K$ when $\tau=0$.

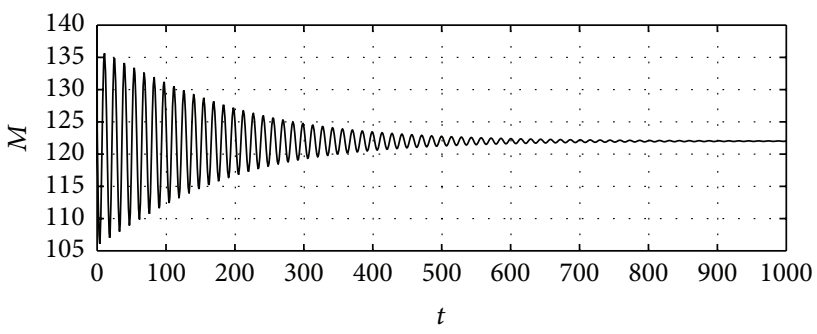

FIgURE 3: History of currency supply $M$ when $\tau=0$.

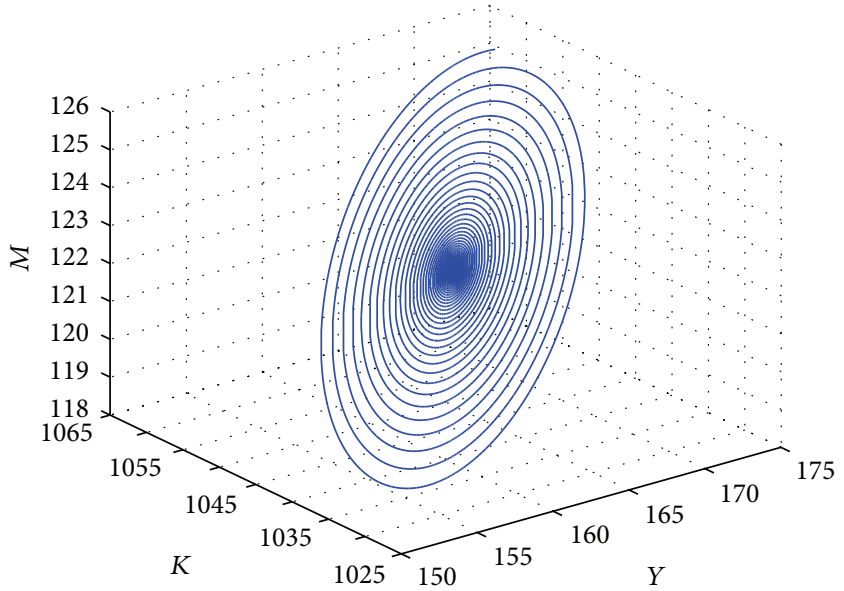

Figure 4: Phase diagram of economic growth system when $\tau=0$.

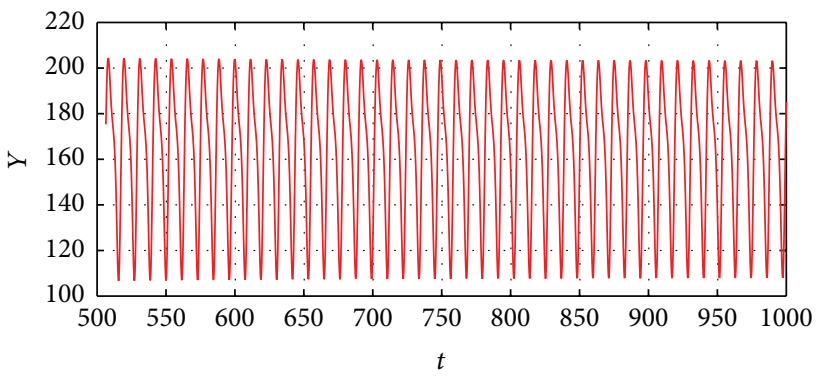

FIGURE 5: History of gross domestic product $Y$ when $\tau=22.045$.

when $\tau>\tau_{0}$, and the bifurcating periodical solution is asymptotically stable. Figures 5-7 show the time histories of the variations, gross domestic product $Y$, physical capital stock $K$, and currency supply $M$ when $\tau=22.045$. Figure 8 shows the phase diagram of economic growth system. We 


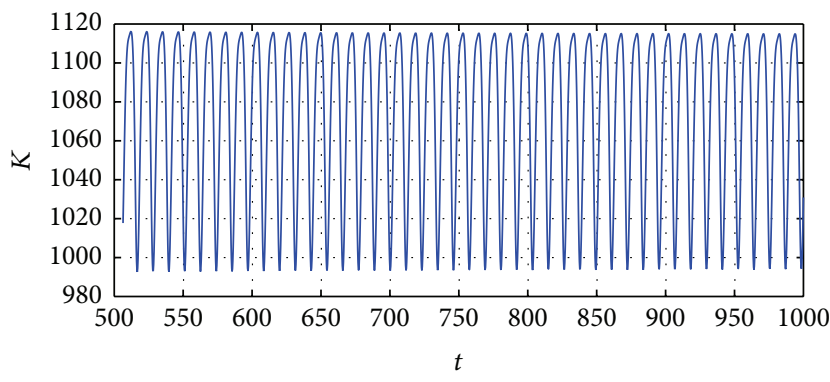

FIgURE 6: History of physical capital stock $K$ when $\tau=22.045$.

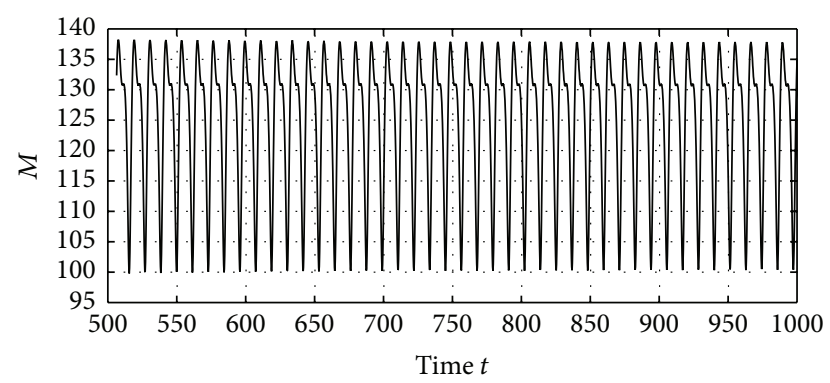

FiguRE 7: History of currency supply $M$ when $\tau=22.045$.

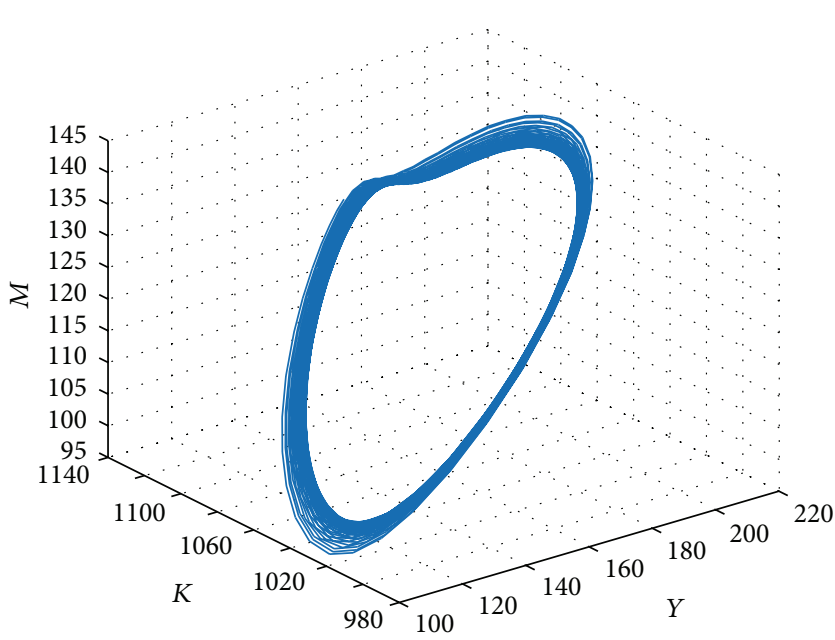

Figure 8: Phase diagram of economic growth system when $\tau=$ 22.045 .

can see clearly that there is a limit cycle near equilibrium $E^{*}(163.6363,1046.3,122)$.

\section{Conclusion}

Nonlinear dynamic finance system model provides rich dynamical behaviors. Whether from the viewpoint of nonlinear system or from implementation of macroeconomic policy, the analysis of the researchers is useful in solving problems of both theoretical and practical importance.

In this paper, based on the research on open Kaldorian business cycle model in [11, 12, 16], a three-dimension business cycle model under the fixed exchange rate condition is established. Firstly, we discuss the existence of equilibrium point and investigate the stability of the system near the equilibrium point with the time delay in currency supply. We find that the system presents different stable status under the different hypothesis, which is shown in Theorem 3. Secondly, we investigate the existence condition of Hopf bifurcation and discuss the stability of periodic solution which is generated by Hopf bifurcation; then, the direction of Hopf bifurcation is given. Furthermore, a numerical simulation is given to confirm the theoretical results.

We conclude that the system will maintain a stable status under the condition that the parameter $\tau$ is in a certain range. Accompanied with the increase of the time delay, the system will lose its stability gradually. Once the parameter $\tau$ is out of the certain range, the system will generate periodical fluctuation; that is, the business cycle is generated. In other words, when the time delay increases to the critical value, the system will undergo Hopf bifurcation and a bifurcation periodical solution will be generated. From our research, the time delay in the money supply in the Kaldorian business cycle model can change the stability of the whole system. The money supply is one of the crucial factors of the business cycle forming. This paper plays an important role in theoretical researching on the model of business cycle with time delay in currency supply, and it is crucial for decision-maker to formulate the macroeconomic policies with the conclusions of this paper.

\section{Competing Interests}

The authors declare that there are no competing interests regarding the publication of this paper.

\section{References}

[1] J. Benhabib and K. Nishimura, "The hopf bifurcation and existence and stability of closed orbits in multisector models of optimal economic growth," in Nonlinear Dynamics in Equilibrium Models, pp. 51-73, Springer, 2012.

[2] X. Zhou, X. Chen, and Y. Song, "Hopf bifurcation of a differential-algebraic bioeconomic model with time delay," Journal of Applied Mathematics, vol. 2012, Article ID 768364, 15 pages, 2012.

[3] J. Ma and H. Tu, "Analysis of the stability and Hopf bifurcation of money supply delay in complex macroeconomic models," Nonlinear Dynamics, vol. 76, no. 1, pp. 497-508, 2014.

[4] N. Kaldor, "A model of the trade cycle," The Economic Journal, vol. 50, no. 197, pp. 78-92, 1940.

[5] M. Kalecki, "A macrodynamic theory of business cycles," Econometrica, vol. 3, no. 3, pp. 327-344, 1935.

[6] M. SzydŁowski and A. Krawiec, "Scientific cycle model with delay," Scientometrics, vol. 52, no. 1, pp. 83-95, 2001.

[7] A. Krawiec and M. Szydlowski, "The KALdor-KALecki business cycle model," Annals of Operations Research, vol. 89, pp. 89-100, 1999.

[8] M. Szydłowski and A. Krawiec, "The KALdor-KALecki model of business cycle as a two-dimensional dynamical system," Journal of Nonlinear Mathematical Physics, vol. 8, supplement1, pp. 266-271, 2001.

[9] A. Kaddar and H. Talibi Alaoui, "Hopf bifurcation analysis in a delayed KALdor-KALecki model of business cycle," Nonlinear 
Analysis. Modelling and Control, vol. 13, no. 4, pp. 439-449, 2008.

[10] L. Wang and X. P. Wu, "Bifurcation analysis of a Kaldor-Kalecki model of business cycle with time delay," Electronic Journal of Qualitative Theory of Differential Equations, no. 27, pp. 1-20, 2009.

[11] M. Lavoie and W. Godley, "Kaleckian models of growth in a coherent stock-flow monetary framework: a Kaldorian view," Journal of Post Keynesian Economics, vol. 24, no. 2, pp. 277-311, 2001.

[12] A. Matsumoto and F. Szidarovszky, "Continuous Hicksian trade cycle model with consumption and investment time delays," Journal of Economic Behavior \& Organization, vol. 75, no. 1, pp. 95-114, 2010.

[13] H.-W. Lorenz, Nonlinear Dynamical Economics and Chaotic Motion, vol. 334, Springer, Berlin, Germany, 1993.

[14] G. Gandolfo, Economic Dynamics: Methods and Models, vol. 16, Elsevier, 1971.

[15] A. Agliari, R. Dieci, and L. Gardini, "Homoclinic tangles in a Kaldor-like business cycle model," Journal of Economic Behavior \& Organization, vol. 62, no. 3, pp. 324-347, 2007.

[16] H.-W. Lorenz, "Analytical and numerical methods in the study of nonlinear dynamical systems in keynesian macroeconomics," in Business Cycles: Theory and Empirical Methods, pp. 73-112, Springer, Berlin, Germany, 1994.

[17] B. D. Hassard, N. D. Kazarinoff, and Y.-H. Wan, Theory and Applications of Hopf Bifurcation, vol. 41, CUP Archive, 1981. 


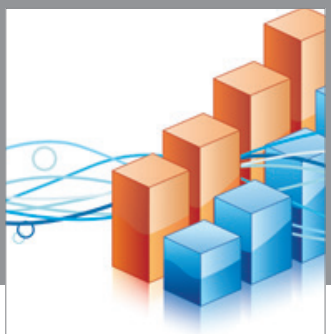

Advances in

Operations Research

vatem alat4

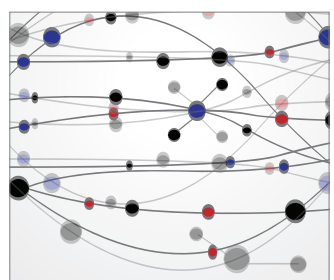

\section{The Scientific} World Journal
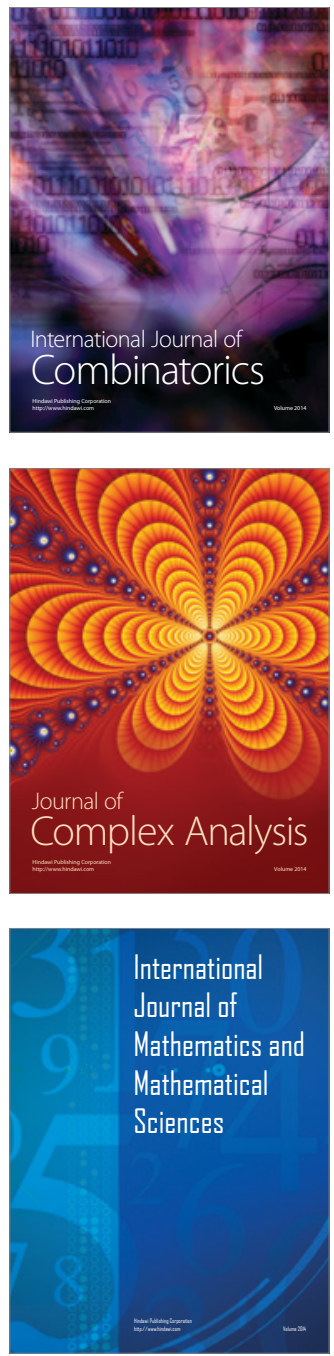
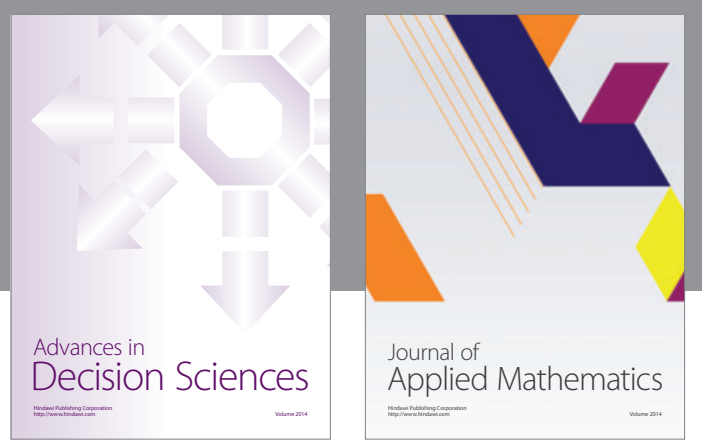

Algebra

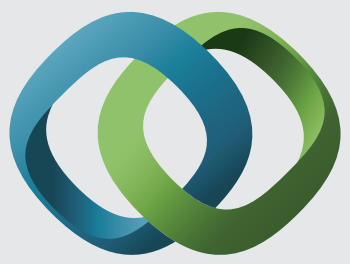

\section{Hindawi}

Submit your manuscripts at

http://www.hindawi.com
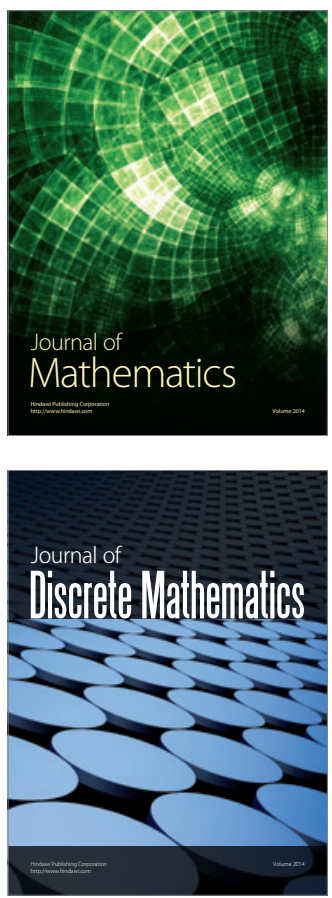

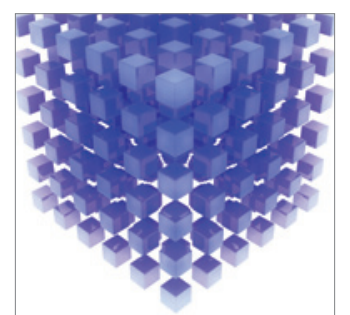

Mathematical Problems in Engineering
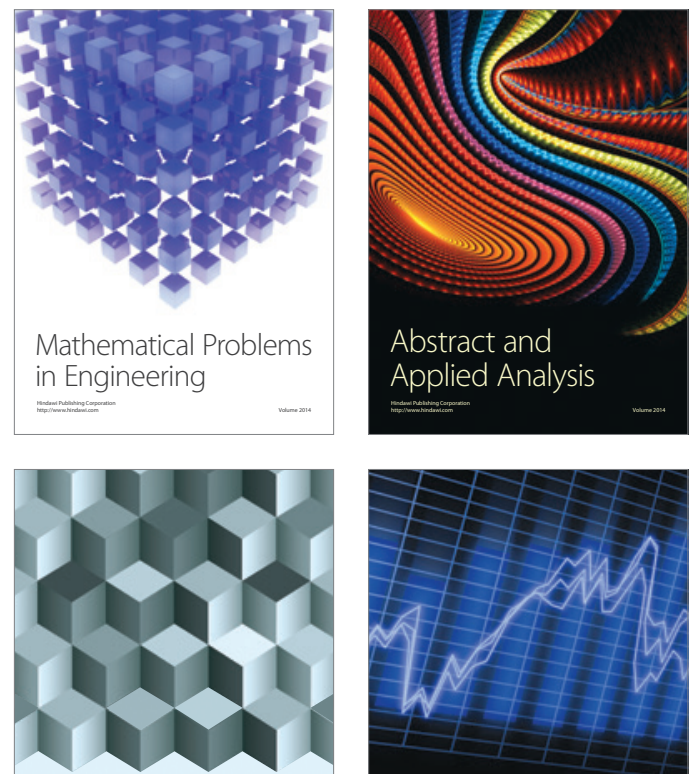

Journal of

Function Spaces

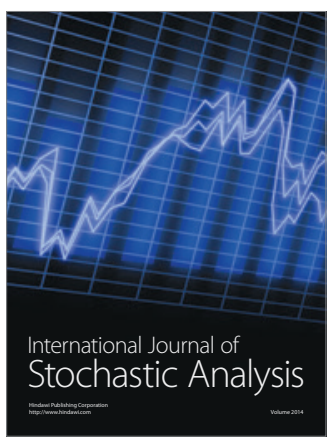

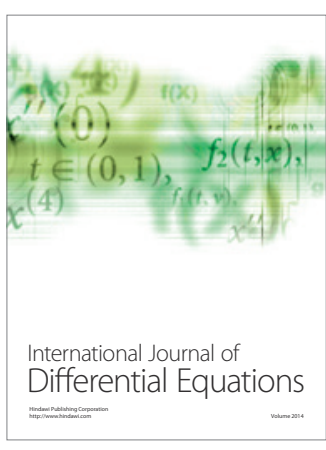
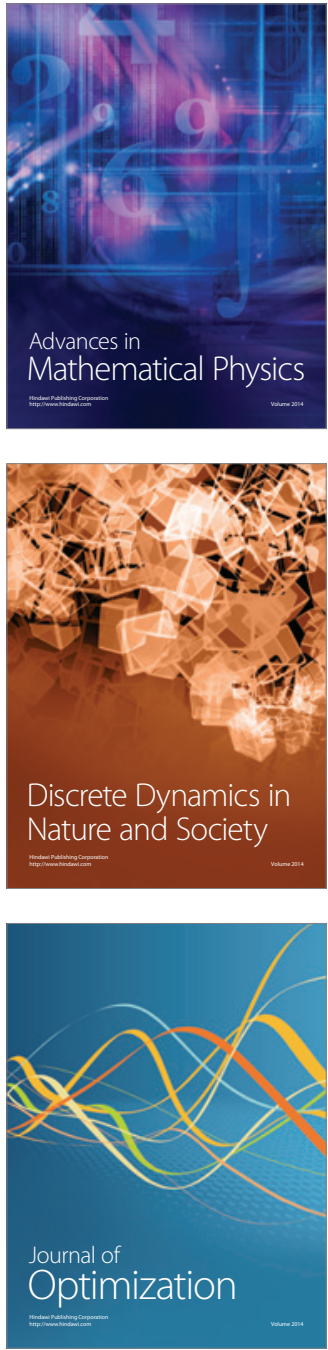